\title{
Cyclic di-GMP acts as a cell cycle oscillator to drive chromosome replication
}

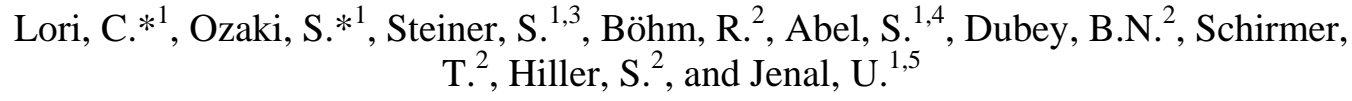
$\mathrm{T}^{2}$, , Hiller, S. ${ }^{2}$, and Jenal, U.,

\begin{abstract}
Affiliations:
${ }^{1}$ Focal area of Infection Biology, Biozentrum, University of Basel

${ }^{2}$ Focal area of Structural Biology and Biophysics, Biozentrum, University of Basel

${ }^{3}$ Current address: Yale University School of Medicine, Boyer Center for Molecular
\end{abstract} Medicine, 295 Congress Avenue, New Haven, CT 06536, USA

${ }^{4}$ Current address: Brigham and Women’s Hospital/Harvard Medical School, Division of Infectious Diseases, 02115 Boston, MA, USA

${ }^{5}$ For correspondence: urs.jenal@unibas.ch

* equal contribution

\section{Key words:}

Two-component system; histidine kinase/phosphatase; phosphorylation cascade; c-diGMP; cell cycle regulation; cell fate determination; replication initiation; cyclin; bac7 teria; Caulobacter crescentus. 
1 Summary:

2 Fundamental to all living organisms is the capacity to coordinate cell division and cell

3 differentiation to generate appropriate numbers of specialized cells. While eukaryotes

4 use cyclins and cyclin-dependent kinases to balance division with cell fate decisions,

5 equivalent regulatory systems have not been described in bacteria. Moreover, the

6 mechanisms used by bacteria to tune division with developmental programs are poor-

7 ly understood. Here we demonstrate that Caulobacter crescentus, a bacterium with an

8 asymmetric division cycle, uses oscillating levels of the second messenger c-di-GMP

9 to drive its cell cycle. We demonstrate that c-di-GMP directly binds to the essential

10 cell cycle kinase CckA to inhibit kinase and stimulate phosphatase activity. An up-

11 shift of c-di-GMP during the G1-S transition switches CckA from the kinase into the

12 phosphatase mode, thereby licensing replication initiation and cell cycle progression.

13 Finally, we show that during division, c-di-GMP imposes spatial control on CckA to

14 install replication asymmetry of future daughter cells. These studies expose c-di-GMP

15 as a cyclin-like molecule in bacteria that coordinates chromosome replication with

16 cell morphogenesis in Caulobacter. The observation that c-di-GMP mediated control

17 is conserved in the plant pathogen Agrobacterium tumefaciens unfolds a general

18 mechanism through which this global regulator of bacterial virulence and persistence

19 coordinates behaviour and cell proliferation. 
1 To enable tissue homeostasis, metazoans tightly regulate the balance between cell

2 proliferation and differentiation ${ }^{1}$. Central players in cell proliferation, development

3 and cell fate decisions are cyclin-dependent kinases (CDKs) ${ }^{2,3}$. To drive cell cycle

4 progression, CDKs associate with oscillating, stage-specific regulatory subunits called

5 cyclins ${ }^{4}$. While in higher organisms cells generally undergo terminal differentiation,

6 bacteria often rely on rapid growth to exploit available nutrients and thus need to dy-

7 namically tune behavioural programs with cell proliferation. How exactly bacteria

8 couple behavioural processes with cell cycle progression remains unclear.

9 A prime model to study the coupling of cell growth and behaviour in bacteria is the 10 aquatic organism Caulobacter crescentus, which strictly separates cell motility from

11 cell proliferation. C. crescentus divides asymmetrically to generate two specialized

12 progeny, a sessile and replication competent stalked and a motile and replication inert

13 swarmer cell. The swarmer cell (G1-phase) re-enters the replication cycle during dif-

14 ferentiation into a stalked cell (S-phase) (Figure 1a). To control the motile-sessile

15 transition, C. crescentus makes use of c-di-GMP, a second messenger controlling a

16 wide range of behavioural processes in bacteria, including virulence, motility and bio-

17 film formation ${ }^{5}$. C-di-GMP levels are low in swarmer cells, increase during differen-

18 tiation to peak in stalked cells and later reach intermediate levels in the predivisional

19 cell $^{6}$. One of the main drivers of c-di-GMP fluctuations is the diguanylate cyclase

20 PleD, which is active in stalked but turned off in swarmer cells ${ }^{7}$ (Figure 1a). While a

21 pleD mutant has reduced levels of c-di-GMP, a strain lacking all diguanylate cyclases

22 (cdG0) is devoid of c-di-GMP ${ }^{6}$. The complete loss of motility and surface attachment

23 in the cdG0 strain illustrates the importance of c-di-GMP oscillation for Caulobacter

24 cell fate determination ${ }^{6}$. In contrast, the role of c-di-GMP in cell cycle progression is

25 unclear. 
1 Our studies originated from a genetic screen for synthetic lethal mutants in the cdG0

2 background. This strain, although viable, shows pronounced morphology and cell cy-

3 cle defects ${ }^{6}$. We thus reasoned that c-di-GMP controls cell cycle progression together

4 with a parallel pathway with partial functional redundancy. The screen revealed a

5 strain with a transposon ( $\mathrm{Tn}$ ) insertion in the promoter region of the gene encoding the

6 single-domain response regulator DivK (PdivK::Tn) (Figure S1a). Crossing back the

7 Tn into the cdG0 mutant produced a strain with severe cell cycle defects (Figure 1b,

8 S1b). This, and the observation that the DNA content per cell mass unit was severely

9 reduced (Figure 1c), indicated that cells are severely compromised for replication ini-

10 tiation. In contrast, growth, division and replication were not affected when the

11 PdivK::Tn was crossed into a $\mathrm{cdG}^{+}$strain (Figure 1b,c). DivK levels were reduced

12 about 10-fold in the cdG0 PdivK::Tn strain (Figure S1c), suggesting that DivK may be

13 limiting for growth. This was confirmed by replacing the divK promoter upstream of

14 the divK gene with the xylose-dependent promoter Pxyl. In the absence of the inducer

15 or in the presence of glucose, which further represses Pxyl activity, DivK levels were

16 strongly reduced compared to wild type (Figure S1d), resulting in severely reduced

17 growth and replication in the cdG0 strain, but not in a $\mathrm{cdG}^{+}$background (Figure 1b,

18 S1e). Together, this indicated that c-di-GMP and DivK convergently regulate cell cy-

19 cle progression.

20 DivK was recently shown to down-regulate the central cell cycle kinase CckA

21 through a direct interaction with DivL, an unorthodox kinase that controls CckA via

22 protein protein interaction ${ }^{8,9}$. CckA initiates a phosphorelay controlling the activity of

23 the response regulator $\mathrm{CtrA}^{10,11}$ (Figure 1a). CtrA is phosphorylated and active in

24 swarmer cells (G1) where it binds to the origin of replication (Cori) to inhibit replica-

25 tion initiation ${ }^{12}$. During differentiation into stalked cells CtrA is inactivated to license 
1 replication initiation ${ }^{13}$. CckA is bifunctional and can act both as kinase and as phos-

2 phatase to control CtrA via the phosphotransfer protein $\mathrm{ChpT}^{14}$. Accordingly, switch-

3 ing CckA from kinase to phosphatase activity during G1-S would rapidly reverse the

4 phosphate flux to inactivate CtrA and authorize replication initiation. Hence, we rea-

5 soned that DivK and c-di-GMP could cooperate to inactivate CtrA. Because c-di-

6 GMP controls CtrA degradation during G1-S transition through the effector protein

$7 \quad$ PopA $^{15}$ (Figure 1a), the G1 arrest of the cdG0 PdivK::Tn strain could conceivably re-

8 sult from simultaneous over-activation and stabilization of CtrA. However, a mutant

9 combining the PdivK::Tn allele with a popA deletion stabilizing CtrA was not affected

10 in growth or DNA replication. In contrast, a PdivK::Tn $\Delta p o p A$ strain that also lacked

11 PleD, produced a strong G1 arrest (Figure 1c, S1f). From this we concluded that c-di-

12 GMP regulates both stability and phosphorylation levels of CtrA during the cell cycle

13 (Figure 1a).

14 To analyse how c-di-GMP regulates CtrA activity, individual components of the

15 CckA-CtrA phosphorelay were purified and examined in vitro. In the absence of c-di-

16 GMP CckA autophosphorylation and phosphotransfer via ChpT to CtrA were readily

17 observed. Strikingly, the addition of c-di-GMP completely abolished phosphorylation

18 of all three components (Figure S2a). When CckA auto-phosphorylation was first car-

19 ried out in the absence of c-di-GMP followed by the addition of c-di-GMP to the reac-

20 tion mixture, rapid dephosphorylation of CckA was observed, arguing that c-di-GMP

21 is a potent stimulator of CckA phosphatase activity (Figure 2a, S2b). Stimulation of

22 the CckA phosphatase was specific to c-di-GMP with GMP, GTP or cGMP having no

23 observable effect (Figure 2a). Experiments with all three components of the phos-

24 phorelay demonstrated that c-di-GMP effectively reverses the phosphate flux of the

25 phosphorelay leading to the inactivation of CtrA (Figure 2b). To test if c-di-GMP also 
1 regulates CckA kinase activity we compared phosphorylation of wild-type CckA with

2 CckA(V366P), a mutant lacking phosphatase activity in vitro (Figure S2c) ${ }^{14}$. When c-

3 di-GMP was added together with $\left[{ }^{32} \mathrm{P}\right] \mathrm{ATP}$ at reaction start, CckA(V366P) phosphor-

4 ylation was strongly reduced as compared to a control lacking c-di-GMP (Figure

5 S2c), indicating that c-di-GMP inhibits CckA kinase activity.

6 These experiments demonstrated that c-di-GMP is a potent trigger to switch CckA

7 from its default kinase into the phosphatase state. Consistent with this, purified CckA

8 specifically binds radiolabeled c-di-GMP (Figure 2c, S2d). Further studies exposed

9 the catalytic ATP-binding domain (CA) as minimal binding region for c-di-GMP

10 (Figure S2e-g). To identify amino acid residues of the CA domain that are specifically

11 involved in c-di-GMP binding, we concentrated on a candidate mutation that was re-

12 cently isolated in the CckA homolog of the plant pathogen Agrobacterium tumefa-

13 ciens $\left(\mathrm{CckA}_{A t}\right)$. In this organism a spontaneous Y674D substitution in the CA domain

14 of $\mathrm{CckA}_{A t}$ was isolated as a motile suppressor of a mutant lacking $\mathrm{PleC}^{16}$. We hypoth-

15 esized that CckA $_{A t}$ is also regulated by c-di-GMP and that in a pleC mutant with ele-

16 vated levels of c-di-GMP (Figure 1a) the Y674D mutation restores its ki-

17 nase/phosphatase balance by interfering with c-di-GMP binding. As shown in Figure

18 S2h, autophosphorylation of purified wild-type CckA $A_{A t}$ was specifically reversed

19 when c-di-GMP was added, while the $\mathrm{CckA}_{A t}(\mathrm{Y} 674 \mathrm{D})$ mutant failed to respond to c-

20 di-GMP. Moreover, c-di-GMP binding to CckA $_{A t}($ Y674D) was strongly reduced as

21 compared to the wild-type form of the protein (Figure S2h). The equivalent substitu-

22 tion in Caulobacter CckA (Y514D) also resulted in strongly diminished c-di-GMP

23 binding (Figure 2c). Importantly, the CckA(Y514D) mutant showed normal kinase

24 activity but failed to dephosphorylate upon addition of c-di-GMP (Figure 2d). This

25 was not due to a general lack of phosphatase activity, as the CckA(Y514D) mutant 
1 showed unaltered basal level phosphatase activity upon ATP depletion (Figure S2i).

2 Together this demonstrated that CckA(Y514D) is compromised for c-di-GMP binding

3 and, as a consequence, cannot switch to the phosphatase mode upon addition of c-di-

4 GMP, resulting in constitutive CckA kinase activity in vitro. To define the c-di-GMP

5 binding pocket on the surface of the CA domain we used a combination of structural

6 modelling, biochemical analysis and NMR spectroscopy (Figures 2e, S2j-l). This ap-

7 proach identified a set of six amino acids, F474, F493, Y514, W523, R537, and F539,

8 which show significant NMR chemical shift perturbations upon c-di-GMP titration

9 experiments and are strictly required for c-di-GMP binding and phosphatase but not

10 kinase activity (Figure 2e, S2i,l). All of these residues locate in close proximity of

11 Y514 in a homology model of CckA (Figure 2e). Interestingly, six of these amino ac-

12 id residues feature aromatic side chains and are well conserved in CckA homologs

13 (Figure S2m). This argues that c-di-GMP is coordinated by the CA domain of CckA

14 via hydrophobic interactions, akin to the binding mode described for the human

15 STING receptor ${ }^{17}$.

16 Next we set out to test if c-di-GMP executes its important cell cycle role primarily by

17 interfering with the CckA kinase/phosphatase balance in vivo. We reasoned that a

18 combination of PdivK::Tn and cckA(Y514D) should cause a similar G1 arrest as ob-

19 served for a strain lacking c-di-GMP altogether. Moreover, this combination should

20 lead to a cell cycle arrest irrespective of the presence of c-di-GMP (Figure S3a). In-

21 deed, cells carrying PdivK::Tn and cckA(Y514D) showed severe growth defects (Fig-

22 ure 3a), increased binding of CtrA to the Cori region (Figure S3b), and a strong G1

23 arrest (Figure 3b, S3c). While this phenotype was independent of PleD, viability of

24 the CckA phosphatase mutant (V366P) strictly depended on c-di-GMP (Figure 3a,b,

25 S3c). This indicated that down-regulation of CckA kinase activity by c-di-GMP is 
1 sufficient to balance the kinase/phosphatase activities of the V366P mutant. To cor-

2 roborate these findings we tested the same cckA alleles in strains expressing $\operatorname{divK}$

3 from the xylose-dependent promoter Pxyl. When Pxyl::divK cells were grown in the

4 absence of xylose, DivK dropped below 10\% of wild type and, as a consequence,

5 cells developed a mild G1 arrest (Figure S3d). This effect was aggravated in strains

6 expressing $c c k A(Y 514 D)$ resulting in a strong reduction of the DNA/cell mass ratio

7 (Figure S3d), severely reduced growth (Figure S3e), increased CckA P phosphoryla-

8 tion levels (Figure S3f) and an overall reduction of the number of chromosomal ori-

9 gins per cell mass (Figure S3g).

10 Taken together, these experiments lead us to propose a model where two convergent

11 regulatory inputs, DivK and c-di-GMP, control the CckA kinase/phosphatase switch

12 to authorize G1-S transition through the inactivation of the replication initiation inhib-

13 itor CtrA (Figure 1a). Intriguingly, cell type specific activity of both PleD and DivK is

14 regulated by DivJ and PleC, two histidine kinase/phosphatase antagonists, which lo-

15 calize to opposite poles of the predivisional cell and during division asymmetrically

16 partition into the daughter cells to determine their respective programs (Figure 1a) ${ }^{18}$.

17 Thus, the two regulators show similar activation profiles during the cell cycle, ${ }^{7,19}$,

18 thereby imposing tight coordination between the DivK branch and the c-di-GMP

19 branch of the CckA switch. This connection is further strengthened by the role of

20 DivK as allosteric activator of the DivJ kinase, a positive feedback mechanism

21 through which both DivK and PleD activity can be rapidly upregulated during G1-S

22 transition $^{19}$. Hence, DivK and c-di-GMP act as molecular connectors between two

23 hierarchical phosphorylation modules, explaining how the cellular dynamics of PleC

24 and DivJ translate into differential activities of the central cell cycle kinase CckA

25 (Figure 4). Because the parallel morphogenetic program critically depends on PleD 
1 activation and the concomitant rise in c-di-GMP concentration ${ }^{6}$, c-di-GMP induced

2 inactivation of CtrA directly couples development to cell cycle progression. This is

3 reminiscent of redundant pathways regulating cell cycle progression in higher eukary-

4 otes, where a multitude of signals converge to control the activity of CDKs ${ }^{20,21}$.

5 In addition to its role in G1-S transition, CckA facilitates cell polarity during division.

6 CckA localizes to both poles of dividing Caulobacter cells but adopts differential ki-

7 nase/phosphatase activities at opposite poles ${ }^{14,22}$ (Figure 1a). The resulting cellular

8 gradient of $\mathrm{Ctr} A \sim \mathrm{P}$ was proposed to establish asymmetric replication activities, which

9 propagate to future daughter cells ${ }^{23}$. To test if c-di-GMP contributes to replication

10 asymmetry during division we made use of fluorescent repressor-operator systems

11 (FROS) to spatially resolve replication initiation events (Figure S4a,b) ${ }^{24}$. While in a

12 majority of wild-type cells chromosome replication originated at the old stalked pole,

13 cells expressing cckA(V366P) or cckA(Y514D) lost replication asymmetry almost en-

14 tirely (Figure S4c). Cells lacking PleD also partially lost their replication preference

15 for the stalked pole. Because active PleD, PleD P, specifically localizes to the stalked

16 pole $\mathrm{e}^{7,25}$, we analysed replicative asymmetry in a cdG0 strain expressing a heterolo-

17 gous diguanylate cyclase, DgcZ, from E. coli, which is uniformly distributed in the

18 cell $^{6,26}$. Although expression of $d g c Z$ restored all developmental defects in this strain ${ }^{6}$,

19 it failed to establish the characteristic spatial replication bias (Figure S4c). From this

20 we conclude that the spatial organization of c-di-GMP metabolism contributes to cell

21 polarity by differentially regulating CckA at opposite cell poles. For example, a local

22 environment with high levels of c-di-GMP might impose CckA phosphatase activity

23 at the stalked pole. Alternatively, a local trough of c-di-GMP may exist at the swarm-

24 er pole with the rest of the cell body containing high levels of c-di-GMP. To distin-

25 guish between these possibilities, we made use of a CckA variant that is unable to lo- 
1 calize to cell poles because it lacks its membrane anchor (cckA $\Delta T M$ ). Expression of

2 this mutant causes massive over-replication and cell filamentation, arguing that delo-

3 calized CckA functions primarily as a phosphatase for $\mathrm{CtrA}^{14}$. In agreement with this,

4 expression of $c c k A \Delta T M(V 366 P)$, lacking phosphatase activity, did not show any ad-

5 verse effects (Figure S4d). Strikingly, expression of $c c k A \Delta T M(Y 514 D)$ in a $\mathrm{cdG}^{+}$

6 strain (Figure S4d) or expression of $c c k A \Delta T M$ in a strain lacking c-di-GMP (Figure

$7 \quad$ S4e) led to a strong G1 arrest, a hallmark of the CckA kinase mode. This indicated

8 that the cellular pool of c-di-GMP strictly imposes phosphatase activity on delocal-

9 ized CckA molecules.

10 Based on these results we propose that the bulk volume of dividing C. crescentus cells

11 experiences high levels of c-di-GMP and that CckA adopts strong kinase activity at

12 the swarmer pole as a consequence of a microenvironment with low levels of c-di-

13 GMP. This view is consistent with the idea that sequestration of CckA to the swarmer

14 pole creates a microenvironment within the cell where CckA can avoid down-

15 regulation by its other inhibitor, DivK $\sim \mathrm{P}^{9,23}$. We propose that CckA sequestration to

16 this subcellular site also shields the protein from the cellular pool of c-di-GMP. Ulti-

17 mately, it is the PleC phosphatase that reduces PleD P and DivK P levels at this sub-

18 cellular site and, possibly together with one or several swarmer pole specific phos-

19 phodiesterases, imposes this spatial regime (Figure 1a, 4). The input from c-di-GMP

20 might also explain how the entire cellular pool of CckA can be tightly regulated.

21 Throughout the cell cycle, CckA localization is often patchy and dynamic without be-

22 ing strictly limited to polar regions ${ }^{22}$. Since the degree of co-localization of DivK and

23 CckA is unclear, c-di-GMP could effectively maintain CckA in the phosphatase state

24 in all cell types or subcellular regions harbouring high levels the second messenger. 
1 C-di-GMP is only one of several novel nucleotide-based second messengers that were

2 recently discovered in bacteria ${ }^{27}$. Their global impact on cell physiology raised the

3 question how these signalling compounds mediate specific cellular responses and how

4 they integrate with other general signalling systems, in particular with two-component

5 phosphorylation networks ${ }^{28}$. Our finding that c-di-GMP acts as a cyclin-like molecule

6 in C. crescentus to control the activity of the cell cycle kinase CckA, establishes the

7 first direct connection between the two most widespread regulatory networks of bac-

8 terial cells. The CckA-ChpT-CtrA pathway is conserved among most known mem-

9 bers of the alpha-proteobacteria, including important pathogens like Bartonella or

10 Brucella ${ }^{29}$. This opens up the exciting possibility that c-di-GMP-imposed control of

11 sensor histidine kinases might represent a general and widespread regulatory mecha-

12 nism in bacteria. Considering that c-di-GMP plays a major role in regulating virulence

13 and persistence, this provides important new entry points into better understanding the

14 behaviour and propagation of bacterial pathogens. 


\section{Acknowledgments}

2 We thank Timothy Sharp from the Biozentrum Biophysics Facility for help with pro-

3 tein analysis and Fabienne Hamburger for plasmid constructions. S.O. is a recipient of

4 a Japan Society for the Promotion of Science (JSPS) Postdoctoral Fellowships for re-

5 search abroad. This work was supported by Swiss National Science Foundation grant

6 310030B_147090 to U.J. and an ERC Advanced Research Grant to U.J.

\section{Author contributions}

9 C.L., S.O., S.A., S.S. and U.J. initiated the project. All authors designed experiments.

10 S.O. carried out genetic experiments. C.L. and S.S. carried out biochemical experi-

11 ments. C.L. and S.O. carried out microscopy experiments. R.B. and S.H. carried out

12 NMR experiments. B.N.D. and T.S. contributed to structural analysis. U.J., S.O., and

13 C.L. wrote the manuscript. 
2 Figure 1: C-di-GMP regulates cell cycle progression via the CckA-CtrA phos3 phorelay.

4 (a) Left: Localization of CckA and factors regulating CckA activity throughout the $C$. 5 crescentus cell cycle. CckA kinase (red) and phosphatase (blue) activities are indicat6 ed. High and low levels of c-di-GMP are shown as grey or white areas, respectively. $7 \quad$ PDE=phosphodiesterase. Right: Regulatory modules inactivating CtrA to control 8 Caulobacter S-phase entry. (b) Growth (left) and cell morphology (right) of strains 9 indicated. 5-fold serial dilutions are shown. Pxyl::divK strains were grown on PYE 10 (none) or PYE glucose (Gluc) plates. (c) Effect of PdivK::Tn on DNA replication in 11 different genetic backgrounds. DNA content and cell mass were determined by flow 12 cytometry. Averages and standard deviations for DNA content/cell mass were ob13 tained from 4 biological replicates. activity.

(a) C-di-GMP specifically stimulates CckA dephosphorylation. CckA phosphorylation reactions were started by adding $\left[{ }^{32} \mathrm{P}\right] \mathrm{ATP}(0 \mathrm{~min})$ and supplemented with c-diGMP $(75 \mu \mathrm{M})$ at the time indicated (arrow). The inlet shows CckA phosphorylation reactions supplemented with c-di-GMP and other nucleotides (75 $\mu \mathrm{M})$. (b) C-di-GMP

21 reverses the phosphate flux of the CckA-ChpT-CtrA phosphorelay. Reactions were run for 30 min and c-di-GMP was added together with $\left[{ }^{32} \mathrm{P}\right] \mathrm{ATP}$ at time $0 \mathrm{~min}(\mathrm{t} 0)$ or

2315 min after reaction start (t15). (c) C-di-GMP binding affinity of CckA. Binding of 24 wild-type CckA (WT) and CckA(Y514D) was determined by UV-crosslinking at in25 creasing concentrations of $\left[{ }^{33} \mathrm{P}\right] \mathrm{c}$-di-GMP (inlet) and quantified as shown in the graph. 
1 Averages and standard deviations were obtained from three replicates. (d) C-di-GMP

2 fails to stimulate phosphatase activity of the CckA(Y514D) mutant. Phosphorylation

3 reactions with wild-type CckA (WT) and Y514D mutant protein were analysed with-

4 out (none) or with c-di-GMP added at time point 0 (t0) or after $15 \mathrm{~min}$ (t15). (e) Ho-

5 mology model of the CA domain of CckA based on a crystal structure of DivL (pdb

$64 q 20)$. Residues that show large $(\Delta \delta(\mathrm{HN})>2$ s.d.) and intermediate $(2$ s.d. $>$

$7 \Delta \delta(\mathrm{HN})>1$ s.d.) amide chemical shift perturbations upon addition of c-di-GMP

8 are shown in purple and pink, respectively. Side chains of residues that contrib-

9 ute to c-di-GMP binding and c-di-GMP mediated phosphatase activity are shown

10 in stick representation and coloured in red. A single molecule of ATP (yellow)

11 was modelled into its putative binding site based on homology to CpxA

12 (pdb4bix). D479, which is involved in ATP binding, is shown green. For more in-

13 formation, see legend to Figures S2k,l.

15 Figure 3: C-di-GMP controls CckA activity to initiate chromosome replication.

16 (a) The $\operatorname{cck} A(Y 514 D)$ allele shows synthetic lethality with PdivK::Tn. 5-fold serial 17 dilutions of strains containing combinations of $c c k A$, PdivK, and pleD alleles were 18 incubated on PYE plates for 2 days. (b) Combining the cckA(Y514D) and PdivK::Tn 19 alleles leads to a G1 arrest. Exponential cultures of mutants containing combinations 20 of $c c k A$, PdivK, and pleD alleles were analysed by flow cytometry. Values of DNA 21 content per cell mass are shown relative to C. crescentus wild type and were obtained 22 as described in the legend for Figure 1c. Averages and standard deviations were ob23 tained from three replicates. 
1 Figure 4: Model of the regulatory circuitry controlling cell cycle progression in 2 C. crescentus.

3 Two intercalated phosphorylation modules control replication initiation through the 4 activity of the replication initiation inhibitor CtrA. When the PleC phosphatase is pre5 sent at the swarmer pole, PleD and DivK are dephosphorylated. In this situation, 6 phosphorylation modules 1 and 2 are uncoupled and CckA adopts DivL-imposed ki7 nase mode to activate CtrA and block replication initiation. When the DivJ sensor ki8 nase is present at the stalked pole, phosphorylation module 1 imposes control on 9 module 2. PleD and DivK are phosphorylated, thereby switching CckA into the phos10 phatase mode and inactivating CtrA. In parallel, c-di-GMP facilitates CtrA degrada11 tion via PopA and the ClpXP protease. 
References:

1. Blanpain, C. \& Simons, B. D. Unravelling stem cell dynamics by lineage tracing. Nat Rev Mol Cell Bio 14, 489-502 (2013).

2. Malumbres, M. Cyclin-dependent kinases. Genome Biol 15, 122 (2014).

3. Ishidate, T., Elewa, A., Kim, S. \& Mello, C. C. Divide and differentiate: CDK/Cyclins and the art of development. Cell Cycle 13, 1384-1391(2014).

4. Morgan, D. O. Cyclin-dependent kinases: Engines, clocks, and microprocessors. Annu. Rev. Cell Dev. Biol. 1-31 (1998).

5. Römling, U., Galperin, M. Y. \& Gomelsky, M. Cyclic di-GMP: the first 25 years of a universal bacterial second messenger. Microbiol. Mol. Biol. Rev. 77, 1-52 (2013).

6. Abel, S. et al. Bi-modal distribution of the second messenger c-di-GMP controls cell fate and asymmetry during the Caulobacter cell cycle. PLoS Genet. 9, e1003744 (2013).

7. Paul, R. et al. Cell cycle-dependent dynamic localization of a bacterial response regulator with a novel di-guanylate cyclase output domain. Genes \& Development 18, 715-727 (2004).

8. Childers, W. S. et al. Cell fate regulation governed by a repurposed bacterial histidine kinase. Plos Biol 12, e1001979 (2014).

9. Tsokos, C. G., Perchuk, B. S. \& Laub, M. T. A dynamic complex of signaling proteins uses polar localization to regulate cell-fate asymmetry in Caulobacter crescentus. Developmental Cell 20, 329-341 (2011).

10. Jacobs, C., Domian, I. J., Maddock, J. R. \& Shapiro, L. Cell cycle-dependent polar localization of an essential bacterial histidine kinase that controls DNA replication and cell division. Cell 97, 111-120 (1999).

11. Biondi, E. G. et al. Regulation of the bacterial cell cycle by an integrated genetic circuit. Nature 444, 899-904 (2006).

12. Quon, K. C., Yang, B., Domian, I. J., Shapiro, L. \& Marczynski, G. T. Negative control of bacterial DNA replication by a cell cycle regulatory protein that binds at the chromosome origin. Proc Natl Acad Sci U S A 95, 120-125 (1998).

13. Domian, I., Quon, K. \& Shapiro, L. Cell type-specific phosphorylation and proteolysis of a transcriptional regulator controls the G1-to-S transition in a bacterial cell cycle. Cell 90, 415-424 (1997).

14. Chen, Y. E., Tsokos, C. G., Biondi, E. G., Perchuk, B. S. \& Laub, M. T. Dynamics of two Phosphorelays controlling cell cycle progression in Caulobacter crescentus. Journal of Bacteriology 191, 7417-7429 (2009).

15. Duerig, A. et al. Second messenger-mediated spatiotemporal control of protein degradation regulates bacterial cell cycle progression. Genes \& Development 23, 93-104 (2009).

16. Kim, J., Heindl, J. E. \& Fuqua, C. Coordination of division and development influences complex multicellular behavior in Agrobacterium tumefaciens. PLoS ONE 8, e56682 (2013).

17. Huang, Y.-H., Liu, X.-Y., Du, X.-X., Jiang, Z.-F. \& Su, X.-D. The structural basis for the sensing and binding of cyclic di-GMP by STING. Nature Structural \& Molecular Biology 19, 728-730 (2012).

18. Wheeler, R., \& Shapiro, L. Differential localization of two histidine kinases controlling bacterial cell differentiation. Molecular Cell 4, 683-694 (1999).

19. Paul, R. et al. Allosteric regulation of histidine kinases by their cognate response regulator determines cell fate. Cell 133, 452-461 (2008). 
20. Choi, Y. J. \& Anders, L. Signaling through cyclin D-dependent kinases. Oncogene 33, 1890-1903 (2014).

21. Hochegger, H., Takeda, S. \& Hunt, T. Cyclin-dependent kinases and cell-cycle transitions: does one fit all? Nat Rev Mol Cell Bio 9, 910-916 (2008).

22. Angelastro, P. S., Sliusarenko, O. \& Jacobs-Wagner, C. Polar localization of the CckA histidine kinase and cell cycle periodicity of the essential master regulator CtrA in Caulobacter crescentus. Journal of Bacteriology 192, 539-552 (2010).

23. Chen, Y. E. et al. Spatial gradient of protein phosphorylation underlies replicative asymmetry in a bacterium. Proceedings of the National Academy of Sciences 108, 1052-1057 (2011).

24. Robinett, C. C. et al. In vivo localization of DNA sequences and visualization of large-scale chromatin organization using lac operator/repressor recognition. The Journal of Cell Biology 135, 1685-1700 (1996).

25. Paul, R. et al. Activation of the diguanylate cyclase PleD by phosphorylationmediated dimerization. J. Biol. Chem. 282, 29170-29177 (2007).

26. Zähringer, F., Lacanna, E., Jenal, U., Schirmer, T. \& Boehm, A. Structure and signaling mechanism of a zinc-sensory diguanylate cyclase. Structure 21, 1-9 (2013).

27. Danilchanka, O. \& Mekalanos, J. J. Cyclic dinucleotides and the innate immune response. Cell 154, 962-970 (2013).

28. Capra, E. J. \& Laub, M. T. Evolution of two-component signal transduction systems. Annu. Rev. Microbiol. 66, 325-347 (2012).

29. Brilli, M. et al. The diversity and evolution of cell cycle regulation in alphaproteobacteria: a comparative genomic analysis. BMC Syst Biol 4, 52 (2010). 

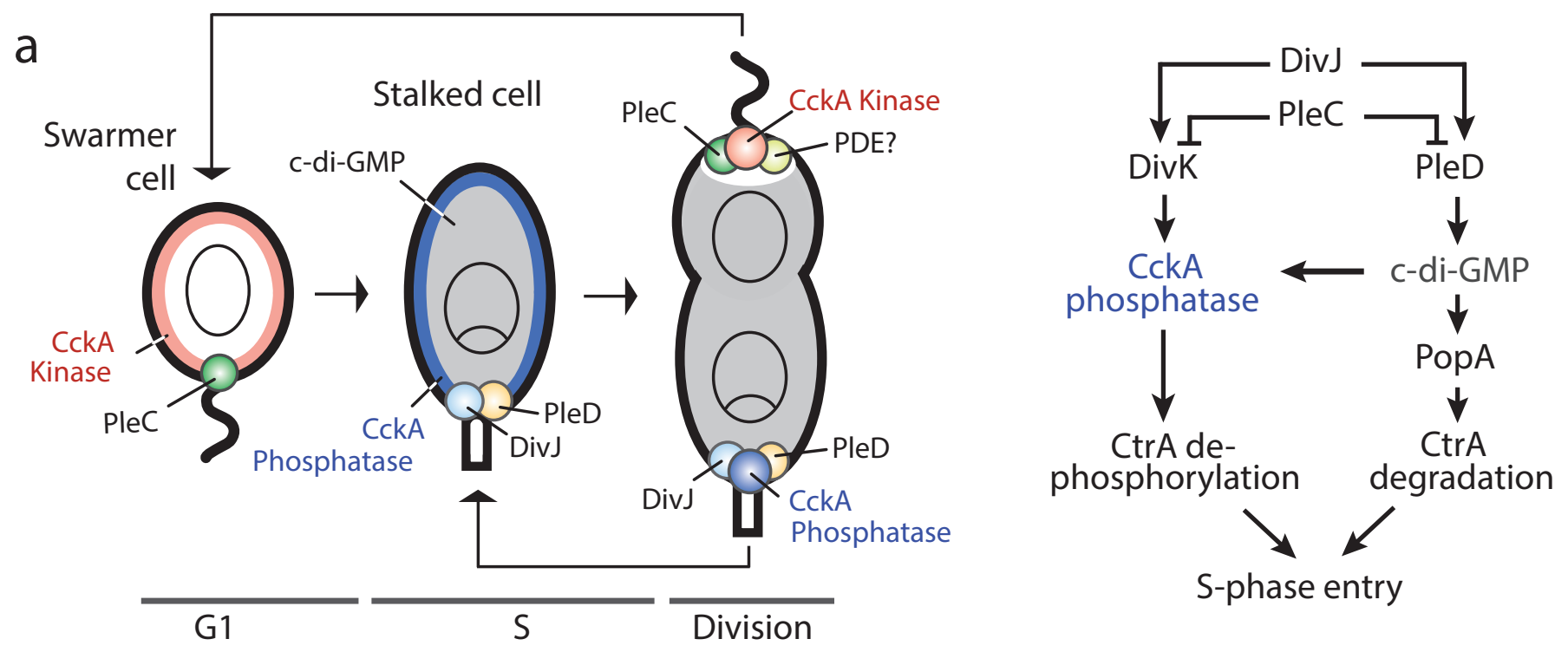

b

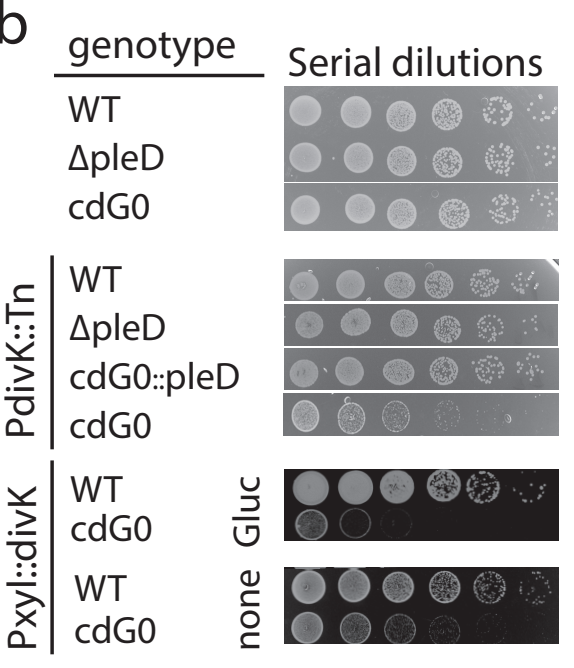

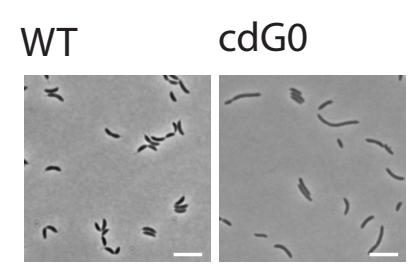

cdGO

PdivK::Tn PdivK::Tn

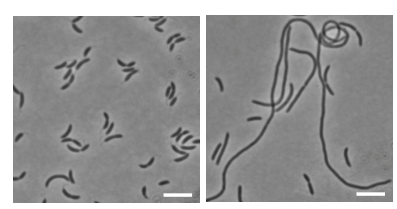

C

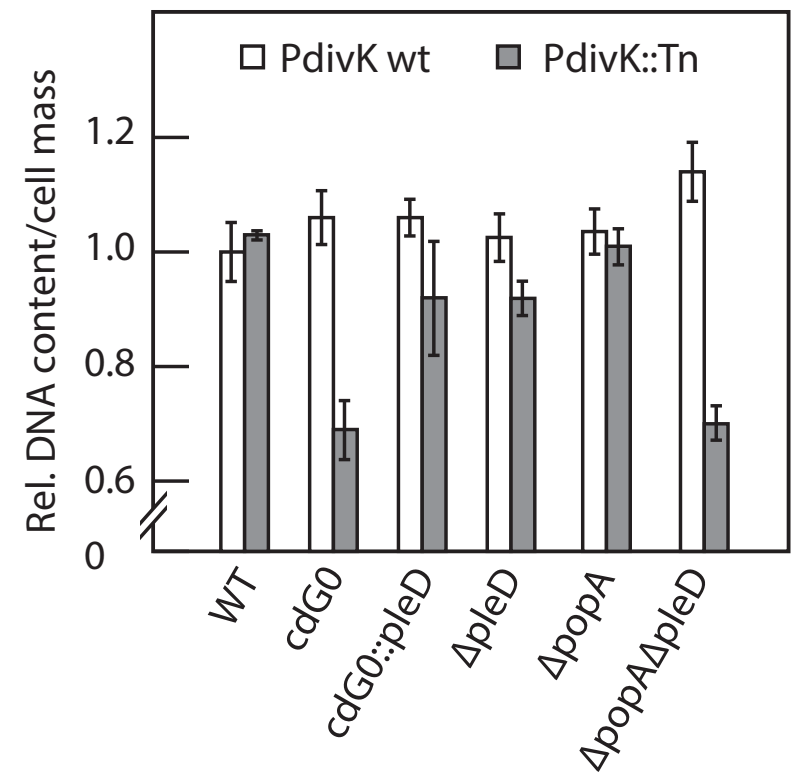



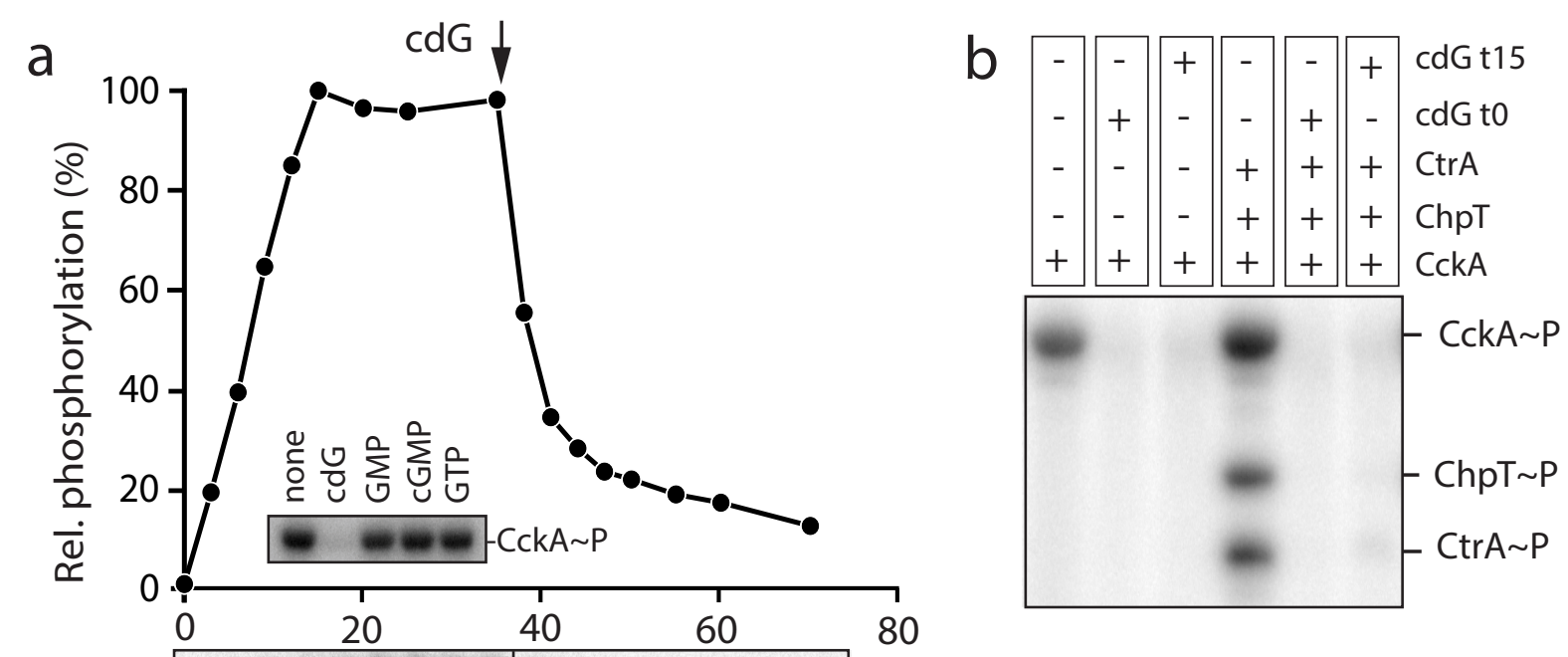

CckA P- \begin{tabular}{|l|l|l|}
\hline time (min) \\
\hline
\end{tabular}
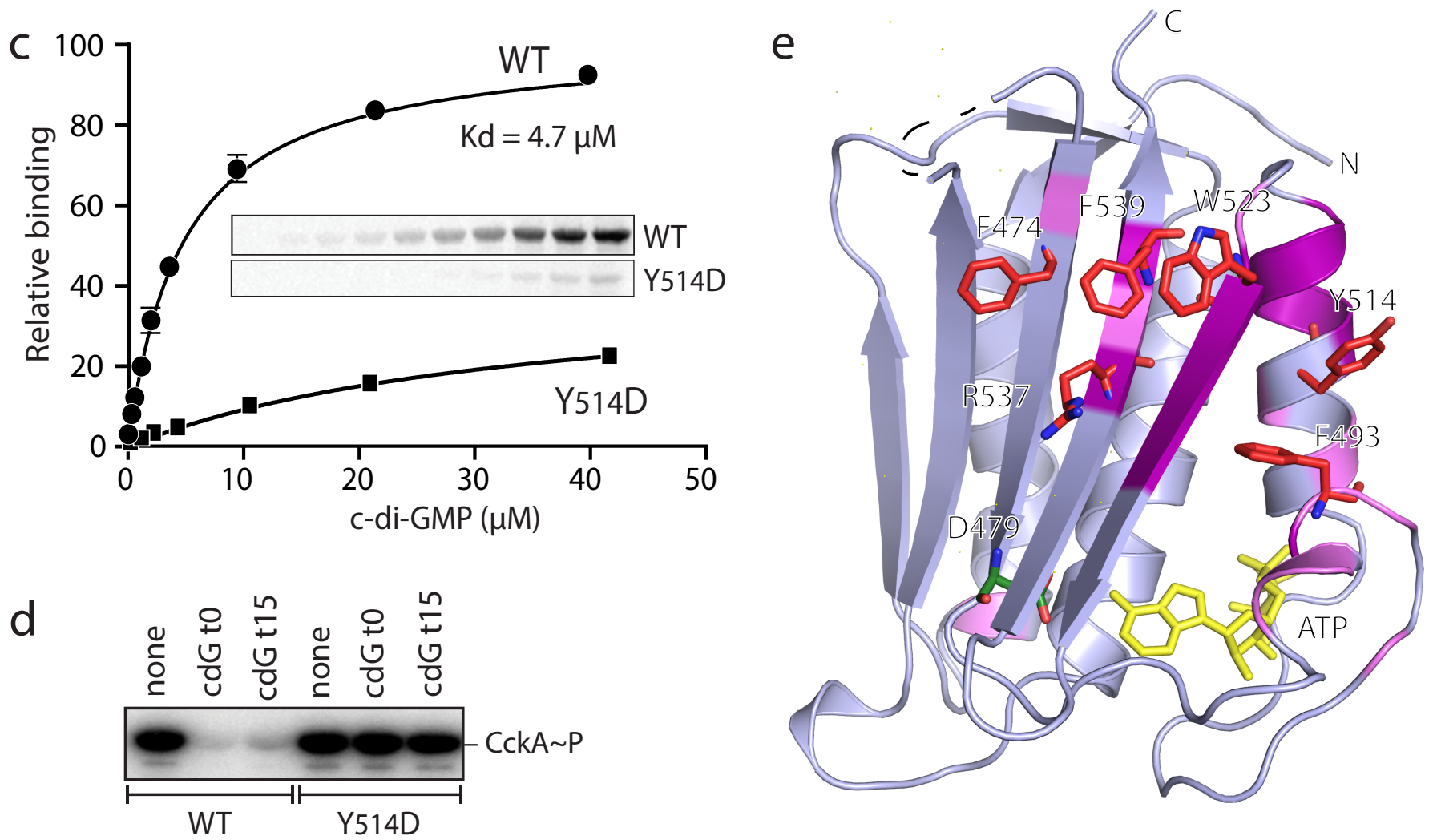


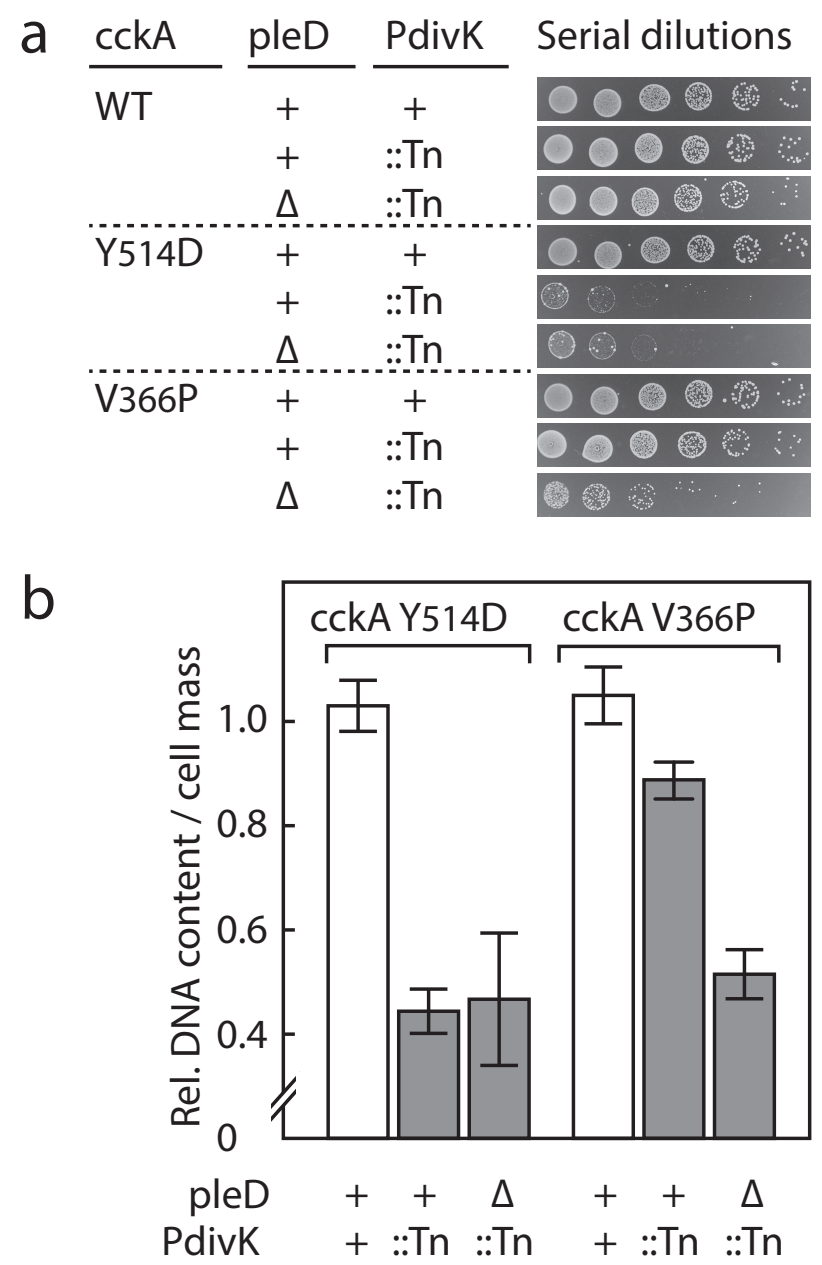


Figure 4

Swarmer cell/pole

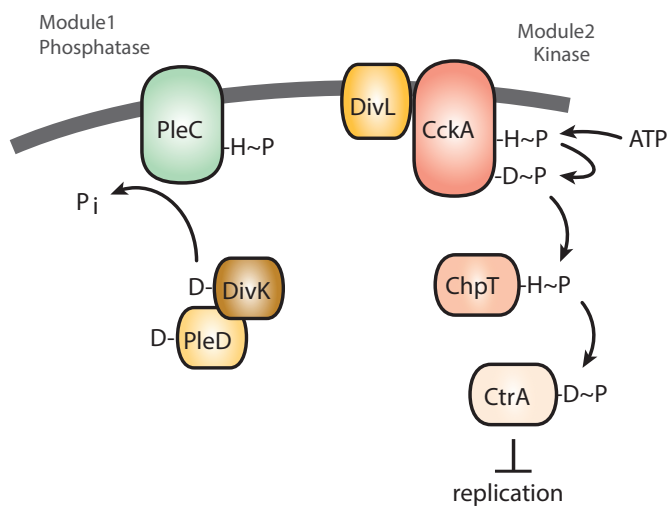

Stalked cell/pole

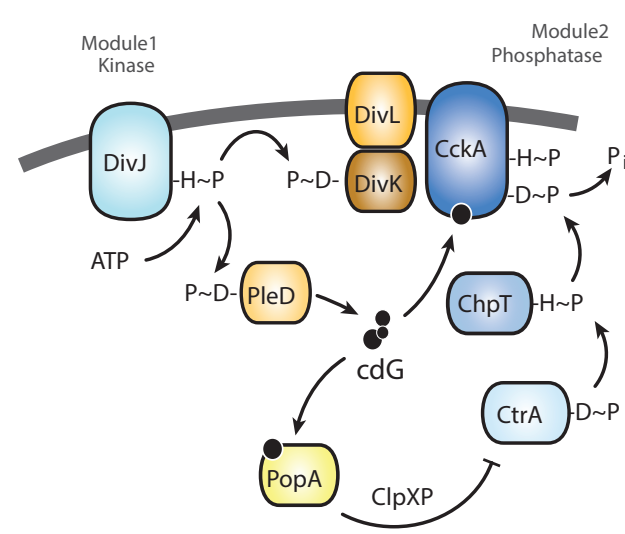

